\title{
Uma Proposta de Rede Social Espontânea para Interação Ubíqua em Eventos
}

\author{
Marcelo da Silva dos $\operatorname{Santos}^{1}$, Cristiano André da Costa ${ }^{1}$, Jorge Luis Victoria \\ Barbosa $^{1}$ \\ ${ }^{1}$ Programa de Pós-Graduação em Computação Aplicada \\ Universidade do Vale do Rio dos Sinos (UNISINOS) - São Leopoldo, RS - Brasil \\ marcelo@novohamburgo.rs.gov.br, \{cac,jbarbosa $\}$ @unisinos.br
}

\begin{abstract}
The popularization of mobile devices and the increasing spread of social networks has fostered the development of applications in order to manage and encourage interactions through their devices. This article presents a model of spontaneous social network to foster the interaction of participants in events such as conferences and congresses. In addition to presenting the model, called Mingle, we show the prototype created for experimentation. The assessment followed two visions, the first focusing on access statistics to the environment and the second checking usability, effectiveness, efficiency and user satisfaction. Results showed good reception Mingle and potential for implementation on a larger scale.
\end{abstract}

Resumo. A popularização dos dispositivos móveis e a crescente difusão das redes sociais fomentou o desenvolvimento de aplicativos com o intuito de gerenciar e incentivar interações através de seus dispositivos. Este artigo apresenta um modelo de rede social espontânea destinada a fomentar a interação dos participantes de eventos, como conferências e congressos. Além de apresentar o modelo, denominado Mingle, mostraremos o protótipo criado para experimentação. A avaliação seguiu duas visões, a primeira focando estatísticas de acesso ao ambiente e a segunda verificando usabilidade, eficácia, eficiência e satisfação dos usuários. Resultados demonstraram boa recepção do Mingle e potencial para implantação em maior escala.

\section{Introdução}

A disseminação dos dispositivos móveis, a evolução tecnológica e os avanços científicos têm aproximado cada vez mais a humanidade do conceito de computação ubíqua [Satyanarayanan 2001] [Beach et al. 2008]. Tais dispositivos fazem parte do cotidiano e acompanham constantemente as pessoas em suas vidas diárias, onde os mesmos são capazes de detecção contínua das atividades e informações espaçotemporais do seu usuário, o que auxilia a compreensão dos contextos onde atua. Portanto, a computação ubíqua [Weiser 1991] aliada à popularização dos dispositivos móveis amplia a possibilidade de exploração de formas de interação ubíqua, ou seja, o conceito de computação integrada ao mundo real, a qualquer momento e em qualquer lugar. Neste sentido, os aplicativos baseados em Serviços Baseados em Localização, que utilizam o contexto de localização do usuário para a recomendação de serviços relacionados a esse local específico [Vaughan-Nichols 2009], tem empregado esforços 
para fornecer serviços que promovam formas de interação espontânea relacionadas à sua localização, tendo as aplicações no formato de redes sociais empregadas ganhado espaço.

A popularização do uso de redes sociais virtuais em camadas cada vez mais jovens da sociedade vem tornando-se parte do cotidiano [Guedes 2013]. Em termos gerais, uma rede social pode ser caracterizada como uma estrutura composta por pessoas ou organizações, essas ligadas por relacionamentos horizontais e não hierárquicos, onde partilham valores e objetivos comuns [Duarte e Frei 2008]. Uma rede social virtual utiliza sistemas automatizados e a Internet para proporcionar esta interação [Costa 2008]. Este trabalho tem como objetivo apresentar o Mingle, um modelo de rede social espontânea [Laforest et al. 2014] [Costa et al. 2014], onde tal rede será formada por usuários fisicamente presentes em um lugar específico, onde as pessoas interagem de forma espontânea sobre um assunto específico de interesse comum, tais como conferências, exposições, galerias, estádios ou restaurantes.

Nesse âmbito, foi desenvolvido um modelo com o intuito de gerenciar e incentivar interações entre usuários através de seus dispositivos móveis, ofertando serviços baseados em localização, com o estabelecimento de uma rede social espontânea. Este poderia ser aplicado a cenários diversos, em aplicações comerciais ou puramente sociais. A partir do modelo, foi criado um protótipo para um congresso que permitiu avaliar a proposta. $\mathrm{O}$ artigo discute a avaliação que considerou aspectos quantitativos e qualitativos do funcionamento do ambiente. A principal contribuição científica do trabalho é permitir a interação entre pessoas de forma ubíqua e espontânea relacionada com contextos atuais. Particularmente, no caso da aplicação o contexto é um congresso e a interação se dá via chat.

Este trabalho está distribuído da seguinte forma: o modelo proposto para a plataforma Mingle é apresentado na Seção 2. A Seção 3 comenta detalhes de implementação e os resultados obtidos e aspectos de interesse desta pesquisa. Na Seção 4 são apresentados mostra de um conjunto de trabalhos relacionados. Finalmente, algumas considerações são feitas na Seção 5.

\section{Modelo Proposto}

Neste trabalho será demonstrado o modelo utilizado na construção da plataforma Mingle [Costa et. al. 2014], um ambiente para fomentar interações sociais espontâneas em ambiente virtual, proporcionando serviços orientados às pessoas participantes da rede presentes dependente da sua localização específica. Neste trabalho a localização de onde o evento está ocorrendo é denominada célula. Os participantes de uma célula podem interagir uns com os outros acessando a plataforma.

A célula pode ser formada para qualquer contexto, como eventos esportivos, concertos, conferências, e assim por diante. A ideia é que pessoas que frequentam o mesmo evento estão envolvidas na mesma atividade. Desta forma, as interações seriam relevantes durante o tempo de duração das atividades e, depois disso, essas pessoas acabariam deixando de compartilhar o mesmo contexto e a rede se desfazendo. A primeira modelagem do ambiente foi sugerida para o uso em conferências e congressos, onde os serviços foram direcionados para este cenário específico. 
A criação de uma nova célula deveria prover, no mínimo, suporte para três requisitos básicos:

- Prover um ambiente de comunicação entre os participantes e palestrantes em congressos e seminários, por meio de salas de comunicação que podem ser criadas pelos próprios participantes;

- Prover um canal para envio de perguntas ao palestrante durante a apresentação, contando com opção para atribuição de pontuação para as perguntas com o intuito de "rankear" as perguntas e organizar a ordem de interesse do grupo;

- Prover um canal para troca de links e conteúdos relacionados à discussão.
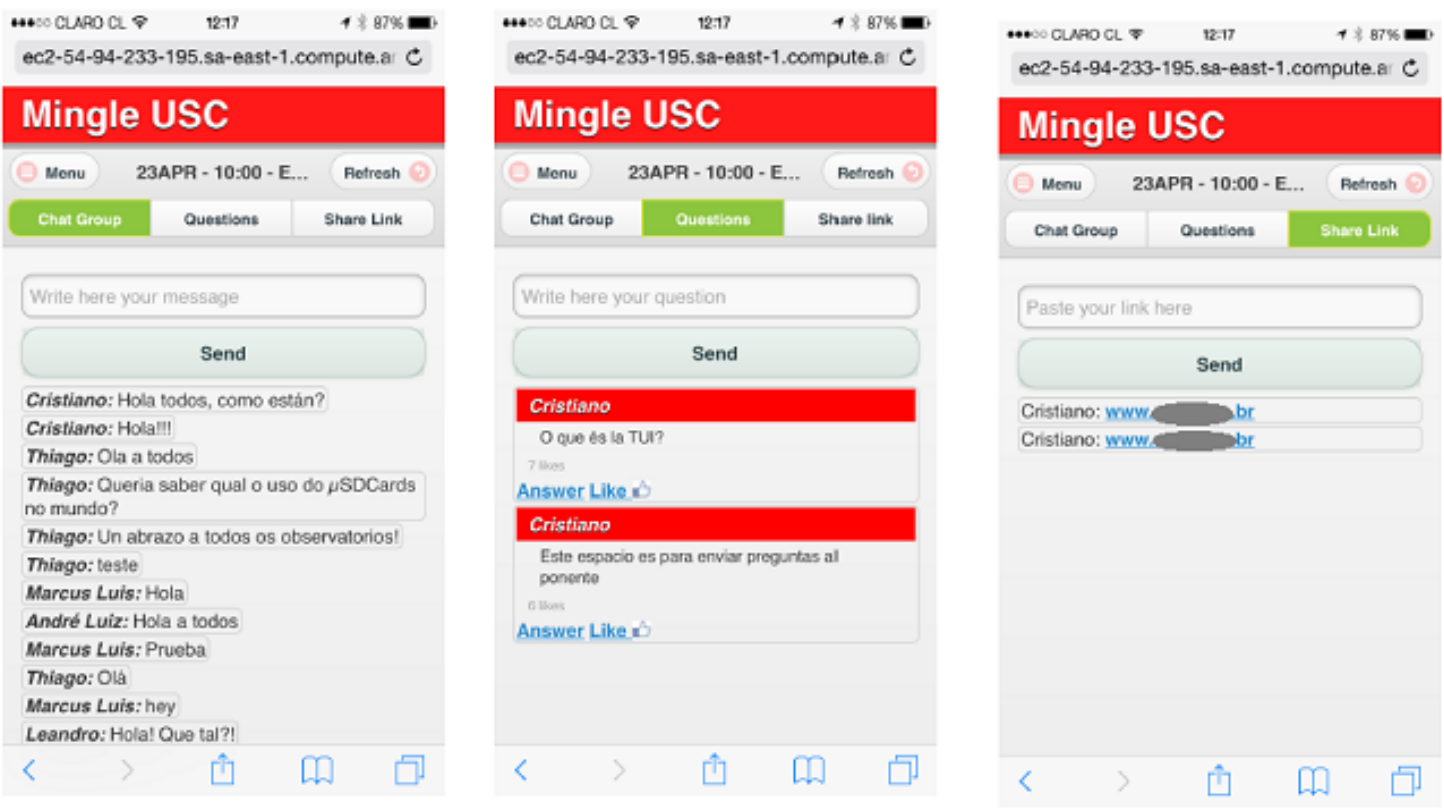

Figura 1. Telas de funcionalidades do Mingle.

Com base nestas informações, foi construído um protótipo inicial com o intuito de validar a proposta. As telas do protótipo com os requisitos acima comentados podem ser vistos na Figura 1. Outros recursos como mapas, agenda, perfil, entre outras, podem ser vistos na Figura 2.

O modelo foi desenvolvido para plataforma web, utilizando o padrão ModelView-Controller (MVC), com módulos comunicando-se via REST através de requisições JSON, permitindo boa comunicação mesmo em situações em que há limitação de recursos e de largura de banda no local. Construído inicialmente como um WebService, esta arquitetura permite futuramente a criação de aplicativos nativos para plataformas móveis (Android, IOS), que podem consumi-lo por meio da API desenvolvida, ampliando o número de sensores e informações disponíveis, visto que poderiam ser utilizados os recursos do dispositivo para a coleta de informações de GPS, por exemplo. 

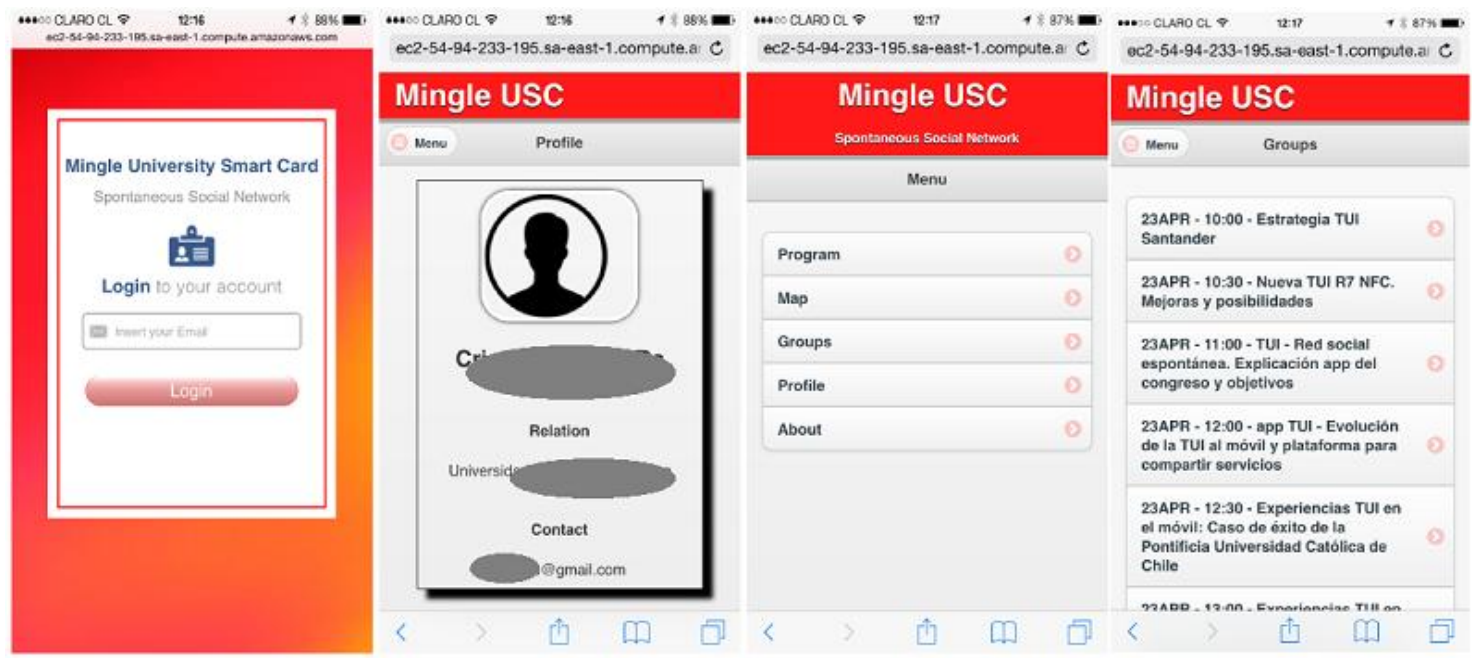

Figura 2. Telas de funcionalidades do Mingle.

A Figura 3 apresenta o fluxo interno do Mingle. O servidor recebe solicitações do usuário (Passo 1), disparando uma requisição ao controlador. Inicialmente, obtemos a requisição com os dados provenientes de um objeto JSON. Através da manipulação deste objeto, é capturado o conteúdo JSON do corpo da requisição e preparada para envio ao banco de dados (Passo 2). Depois de avaliado se o pedido foi de uma consulta (agenda, pesquisa...) (Passo 3-a) ou uma entrada no chat da célula (Passo 3-b), ela é enviada para a base de dados respectiva.

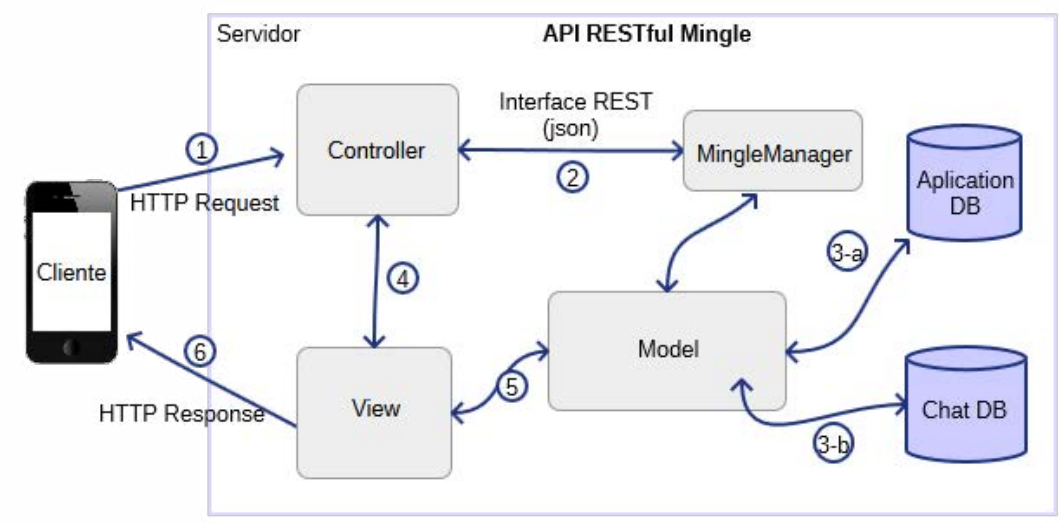

Figura 3. Arquitetura do Mingle e principais interações entre os componentes.

\section{Avaliação e Discussão}

O experimento foi realizado durante congressos em duas edições. Os dados foram colhidos durante as duas edições do Congresso Internacional dedicado ao TUI (Cartão Universitário Inteligente), evento destinado a análise de tendências, situação e avanço na utilização do TUI no mundo, ambos ocorridos no Chile, a primeira edição compreendida entre os dias 23 e 24 de abril, doravante denominado de Célula 1 e a segunda edição ocorrida no dia 3 de dezembro, doravante denominado de Célula 2, ambos no ano de 2015. Os eventos apresentaram um número expressivo de participantes recorrentes, característica que foi utilizada para avaliação da proposta. Foram colhidos como grupo 
amostral aqueles usuários que participaram das duas edições e que preencheram a pesquisa nas duas ocasiões.

A avaliação segue duas abordagens, a primeira focando quantitativamente os números de acesso ao ambiente através das estatísticas geradas pela utilização. A segunda foi uma abordagem qualitativa através de aplicação de questionário junto aos usuários. A primeira etapa de avaliação foi executada por meio do uso de ferramentas digitais para mensuração do conteúdo produzido e volume de interações nos conteúdos propostos. A segunda focou em um nível de realidade que não pode ser quantificado, trabalhando com o universo de significados, das ações, motivos, crenças, aspirações, valores, atitudes e relações humanas, captadas a partir do olhar dos pesquisadores, ou seja, preocupa-se principalmente em compreender e explicar a dinâmica das relações sociais [Minayo 2007]. Para sua avaliação, foi aplicado um questionário direcionado para investigar aspectos como usabilidade, confiabilidade, atendimento as expectativas, pontos negativos e positivos, dentre outras questões.

\subsection{Avaliação quantitativa}

Para obtenção das métricas de acesso e tráfego no ambiente foram extraídas estatísticas com auxílio da ferramenta Google Analytics (2016). Os dados foram colhidos durante os dias que ocorreram os congressos. Dentre os dados levantados na primeira aferição da ferramenta, no primeiro dia da Célula 1 foi registrado um pico de acessos de 4.255 visualizações de páginas em um único dia, com um tempo médio de 18 minutos e 35 segundos por sessão e que esta média aumentou para 22 minutos e 53 segundos no segundo dia. Nas leituras posteriores notou-se que o número estabilizou na média de 3.100 visualizações durante os quatro dias que se sucederam ao término do evento.

Referente ao comportamento dos usuários seguem números obtidos na primeira e segunda edição do evento:

- Na Célula 1 o ambiente contava com 127 usuários ativos, usuários que apresentavam um mínimo de dois acessos durante o período em que a extração de dados foi efetuada. Em um total de 149 participantes cadastrados temos um percentual de $85,23 \%$ de adesão;

- Na Célula 2 o ambiente contava com 84 usuários ativos, usuários que apresentam um mínimo de dois acessos durante o período em que a extração de dados foi efetuada. Em um total de 164 participantes cadastrados temos um percentual de $51,22 \%$ de adesão;

Referente ao percentual de tempo de permanência por sessão, aqueles que realizaram autenticação e realizaram interações com o ambiente, seguem números obtidos na primeira e segunda edição do evento:

- Na Célula 1 foi registrado um tempo médio de 20 minutos e 44 segundos por sessão, com uma média de 10 autenticações durante o período avaliado, com tempo médio de 1 minuto e 22 segundos por página visualizada;

- Na Célula 2 foi registrado um tempo médio de 26 minutos e 47 segundos por sessão, com uma média de 12 autenticações durante o período avaliado, com tempo médio de 2 minutos e 56 segundos por página visualizada; 
Referente ao número de mensagens trocadas somadas todas as interações com o ambiente, seguem números obtidos na primeira e segunda edição do evento:

- Na Célula 1 foram registradas 106 mensagens trocadas, somando intervenções no chat, troca de links e perguntas/respostas das palestras;

- Na Célula 2 foram registradas 245 mensagens trocadas, somando intervenções no chat, troca de links e perguntas/respostas das palestras;

Os dados demonstraram que a plataforma, em um primeiro momento recebeu um grande número de participantes que efetuaram apenas leituras rápidas as mensagens, sem interação com a ferramenta. Notou-se uma diferença significativa no decorrer no tempo, onde $78 \%$ das mensagens enviadas foram do final do primeiro dia até o término do evento. Neste primeiro contato, as mensagens, em sua maioria, eram para testar o ambiente e conforme a confiança aumentou, os conteúdos passaram a ser relevantes e relacionados aos temas propostos durante as palestras.

Comparando as duas Células, na primeira nota-se um número maior de adesões, levando em consideração o número de inscritos no evento comparado ao número de usuários ativos. No entanto, durante a ocorrência da Célula 2 notou-se um número menor de adesões, mas um aumento no tempo de permanência e número de mensagens trocadas.

\subsection{Pesquisa Qualitativa}

A segunda estratégia de avaliação foi convidar os usuários da plataforma a responderem um formulário de pesquisa com o intuito de conhecer as impressões dos mesmos no uso da ferramenta. O mesmo questionário foi aplicado nas duas Células e com ele buscou-se verificar aspectos tanto da ferramenta, como usabilidade e navegabilidade, como suas expectativas, uso da interface e visão de futuros trabalhos aplicando os recursos apresentados. Para manter equilíbrio no grupo amostral, foi selecionado um grupo de 20 usuários que tenha respondido ambas as edições do evento.

O questionário aplicado para esta avaliação esteve disponível no próprio ambiente em uma das opções do menu principal. Essa avaliação seguiu o modelo de aceitação tecnológica (TAM) proposto por Davis (1989) e ampliado por Yoon e Kim (2007), usando escala de Likert (1932). Conforme Davis (1989), dentre os vários fatores que os usuários podem considerar importantes para aceitar ou rejeitar um aplicativo o primeiro deles é a percepção de sua utilidade, ou seja, se ele consegue notar melhora ou ganhos na sua experiência na medida em que ele o utiliza. O segundo fator que pode influenciar é quanto a de facilidade na utilização, a qual é definida como o grau de facilidade com que as pessoas podem empregar uma ferramenta ou objeto a fim de realizar uma tarefa específica e importante. Este critério complementa o primeiro, porque de acordo com pesquisas [Yoon e Kim 2007], fornecer um aplicativo com boa usabilidade não significa que esse será útil para o usuário, ou que os benefícios da aplicação justifiquem uma alta complexidade no seu uso.

Para avaliação, os usuários foram convidados a selecionar uma das opções na escala proposta. Os critérios foram definidos como: Discordo, Discordo parcialmente, Indiferente, Concordo parcialmente e Concordo. 
Tabela 1. Resultados da avaliação para o item Percepção de Utilidade

\begin{tabular}{|c|c|c|c|c|c|}
\hline Questão & Concordo & $\begin{array}{l}\text { Concordo } \\
\text { parcialmente }\end{array}$ & Indiferente & $\begin{array}{c}\text { Discordo } \\
\text { parcialmente }\end{array}$ & Discordo \\
\hline 1. As opções do menu são relevantes. & $85,7 \%(18)$ & $14,3 \%(3)$ & $0 \%(0)$ & $0 \%(0)$ & $0 \%(0)$ \\
\hline $\begin{array}{l}\text { 2. O App facilita a interação com o } \\
\text { apresentador. }\end{array}$ & $76,2 \%(16)$ & $19 \%(4)$ & $0 \%(0)$ & $4,8 \%(1)$ & $0 \%(0)$ \\
\hline $\begin{array}{l}\text { 3. O App estimula a discussão de temas } \\
\text { apresentados. }\end{array}$ & $85,7 \%(18)$ & $14,3 \%(3)$ & $0 \%(0)$ & $0 \%(0)$ & $0 \%(0)$ \\
\hline $\begin{array}{l}\text { 4. O App facilita as interações em uma } \\
\text { conferência. }\end{array}$ & $81 \%(17)$ & $19 \%(4)$ & $0 \%(0)$ & $0 \%(0)$ & $0 \%(0)$ \\
\hline $\begin{array}{l}\text { 5. Gostaria de usar o App em eventos na } \\
\text { minha universidade ou empresa, se } \\
\text { disponível. }\end{array}$ & $76,2 \%(16)$ & $19 \%(4)$ & $4,8 \%(1)$ & $0 \%(0)$ & $0 \%(0)$ \\
\hline
\end{tabular}

$\mathrm{Na}$ Tabela 1, são apresentadas as questões que abordam a percepção de utilidade da ferramenta em relação ao uso na comunicação durante o decorrer das palestras e atividades. Na Tabela 2, podemos ver as questões que abordam a interface da ferramenta avaliando as características de usabilidade. Para embasar a avaliação foram aplicados os conceitos de Nielsen (2014), apresentando a cada questão os parâmetros de avaliação e a definição de cada quesito.

Tabela 2. Resultados da avaliação para o item Facilidade de Utilização

\begin{tabular}{lccccc}
\hline Questão & Concordo & $\begin{array}{c}\text { Concordo } \\
\text { parcialmente }\end{array}$ & Indiferente & $\begin{array}{c}\text { Discordo } \\
\text { parcialmente }\end{array}$ & Discordo \\
\hline 1. O aplicativo é fácil de entender. & $61,9 \%(13)$ & $33,3 \%(7)$ & $4,8 \%(1)$ & $0 \%(0)$ & $0 \%(0)$ \\
2. O aplicativo é fácil de usar. & $57,1 \%(12)$ & $42,9 \%(9)$ & $0 \%(0)$ & $0 \%(0)$ & $0 \%(0)$ \\
3. A navegação nas opções é simples. & $47,6 \%(10)$ & $42,9 \%(9)$ & $4,8 \%(1)$ & $0 \%(0)$ & $4,8 \%(1)$ \\
$\begin{array}{l}\text { 4. Com pouco esforço, eu posso interagir } \\
\text { em um grupo usando o bate-papo. }\end{array}$ & $95,2 \%(20)$ & $4,8 \%(1)$ & $0 \%(0)$ & $0 \%(0)$ & $0 \%(0)$ \\
$\begin{array}{l}\text { 5. Com pouco esforço, posso fazer uma } \\
\text { pergunta em um grupo. }\end{array}$ & $85,7 \%(18)$ & $14,3 \%(3)$ & $0 \%(0)$ & $0 \%(0)$ & $0 \%(0)$ \\
$\begin{array}{l}\text { 6. Com pouco esforço, eu posso } \\
\text { compartilhar um link. }\end{array}$ & $66,7 \%(14)$ & $14,3 \%(3)$ & $19 \%(4)$ & $0 \%(0)$ & $0 \%(0)$ \\
7. As opções são claras e objetivas. & $61,9 \%(13)$ & $38,1 \%(8)$ & $0 \%(0)$ & $0 \%(0)$ & $0 \%(0)$ \\
\hline
\end{tabular}

Também foi apresentada uma questão dissertativa aos avaliadores: "Quais suas sugestões para possamos efetuar melhorias na próxima versão do Mingle?". Comparando as respostas, notou-se que $80 \%$ dos participantes acreditam que a proposta incrementou a comunicação entre os usuários durante os eventos, não só aumentando a rede de disseminação de conhecimento e colaboração, mas como ferramenta para discussão de projetos correlacionados. Este número não teve variação significativa entre os eventos. 
Outras respostas mostraram impressões positivas também quanto à aparência do ambiente e a aplicação dos recursos para colaboração, pelo canal para trocar experiências e compartilhar informações. Em contraponto, um pequeno número apontou que não vislumbrava a utilidade para auxilio das praticas e que não utilizaria em outros eventos. Outros relataram questões de desempenho na atualização das mensagens.

\section{Trabalhos relacionados}

Nesta seção são apresentados alguns trabalhos que empregam conceitos de rede social aplicada ao campo da computação móvel e ubíqua. Entre aqueles, foi considerado o mais representativo e também os modelos que tiveram alguma espontaneidade, ou seja, criar redes sociais relacionadas a um contexto específico.

O projeto C3PO [Laforest et al. 2014] propõe um modelo para troca de conteúdo multimídia em redes sociais que denominam Redes Sociais Espontâneas e Efêmeras (SESNs). Eles contam com uma arquitetura peer-to-peer distribuída formada espontaneamente por dispositivos móveis de participantes do evento. As SESN podem ser criadas por qualquer usuário e anunciado para outros usuários nas imediações. Estes serviços são descobertos invocando técnicas de redes oportunistas. Confirmando participação, eles serão capazes de comentar as apresentações, compartilhar fotos durante a conferência e os eventos sociais, votar na melhor apresentação ou demonstração, entre outras atividades.

Em [Sancho-Asensio et. al 2014] é apresentado um framework orientado à formação de grupos em ambientes de Aprendizagem Colaborativa, efetuando agrupamentos de alunos com base em seus perfis. Como motivação para realizar o agrupamento apresenta o objetivo de fomentar o trabalho colaborativo dos alunos.

Tourist-MSN [Arnaboldi et. al. 2014] projetados para melhorar experiências durante visitas turísticas, permitindo que os indivíduos possam criar, coletar $\mathrm{e}$ compartilhar informações úteis, através de mensagens assíncronas ou por chat em tempo real, relacionadas a pontos de visitação turística. A aplicação utiliza CAMEO (ContextAware Middleware for Opportunistic Mobile Social Networks), plataforma para dispositivos móveis capaz de fornecer um conjunto de ferramentas para $o$ desenvolvimento de serviços e aplicações colaborativas (baseado em comunicações oportunistas) com alto impacto social [Arnaboldi et. al. 2011]. Sendo assim, ela fornece um conjunto de funcionalidades comuns a redes sociais móveis com base na coleta eficiente, modelagem e inferência de informações de contexto multidimensional, que visam reconhecer a localização do usuário e fornecer a ele informações pertinentes.

\section{Considerações finais}

Com a influência da Web sobre todas as facetas da vida contemporânea, vidas virtual e real estão cada vez mais se integrando, criando novas oportunidades para o compartilhamento de informações, desenvolvimento de aplicações que exploram os conceitos de sensibilidade ao contexto e as redes sociais. Neste trabalho, apresentamos o protótipo criado para uma rede social espontânea, denominado Mingle, e realizamos a sua avaliação tendo em mente a ideia de fomentar o compartilhamento de informações e conteúdos que estão relacionados com locais específicos. 
O protótipo foi construído como um webservice utilizando o padrão MVC, com módulos comunicando-se via RESTful, permitindo boa comunicação mesmo em situações em que há limitação de recursos e de largura de banda no local. Esse recurso é especialmente interessante quando o cliente utilizado se tratar de smartphones ou tablets, considerando capacidade limitada em termos de poder de processamento e bateria.

Além disso, o cenário desenvolvido permite a criação de aplicativos nativos para plataformas móveis (Android, IOS) consumindo o webservice por meio da API desenvolvida, neste caso ampliando o número de sensores e informações disponíveis para calibragem do perfil.

A avaliação discutida nesse artigo mostrou uma perspectiva positiva sobre a proposta, mostrando contentamento e facilidade no uso das ferramentas disponibilizadas aos participantes mesmo sendo tímidas as contribuições à produção de conteúdo, mas, ainda assim, dentro de uma faixa esperada nesse período de adaptação.

Como trabalho futuro, pretendemos expandir o modelo com o gerenciamento automatizado dos contextos [WAGNER, BARBOSA e BARBOSA 2014], permitindo um compartilhamento de informações entre diferentes células, a respeito do usuário e seu histórico. Finalmente, pretendemos aumentar a avaliação da plataforma Mingle em um ambiente real e testar o servidor com cargas geradas a partir desse uso.

\section{Agradecimentos} pesquisa.

Os autores gostariam de agradecer ao Santander Universidades pelo apoio à essa

\section{Referências}

Arnaboldi, V.; Conti, M.; Delmastro, F. (2011) "Implementation of CAMEO: a contextaware middleware for opportunistic mobile social networks", IEEE Int. Symposium World of Wireless, Mobile and Multimedia Networks, IEEE Computer Society, pp. $1-3$.

(2014) "Cameo: a novel context-aware middleware for opportunistic mobile social networks", Pervasive and Mobile Computing, vol. 11, Elsevier, pp. 148-167.

Beach, A., Gartrell, M., Akkala, S., Elston, J. et al. (2008) "WhozThat? evolving an ecosystem for context-aware mobile social networks", IEEE Network, vol. 22, no. 4, pp. 50-55.

Costa, C., Yamin, A. e Geyer, C. (2008) "Toward a general software infrastructure for ubiquitous computing”, IEEE Pervasive Computing, vol. 7, pp. 64-73.

Costa, C. A., Zaupa, D., Barbosa, J. L. V., Righi, R. D. R., Camargo, J. Daroit, N., Yamin, A. C. (2014) "A spontaneous social network based on mobile devices", Social Network Analysis and Mining, vol. 4, no. 1, pp. 158.

Davis, F.D. (1989) "Perceived usefulness, perceived ease of use, and user acceptance". MIS Q 319-340

Duarte, Fábio, Frei, Klaus. (2008) "Redes Urbanas. Redes Urbanas”, In: Duarte, Fábio; Quandt, Carlos; Souza, Queila. O Tempo Das Redes, p.156. 
Google Analitics. (2016) "Gráficos de acesso e utilização". Disponível em: $<$ https://accounts.google.com/>.

Guedes, Taís Morais. (2013) "As Redes Sociais — Facebook e Twitter — e suas influências nos Movimentos Sociais", Monografia de especialização. Universidade de Brasília. Brasília.

Laforest, F. et al. (2014) "C3PO: a Spontaneous and Ephemeral Social Networking Framework for a collaborative Creation and Publishing of Multimedia Contents", In MoWNet 2014, pages 1-6, Rome, Italy, Elsevier.

Likert, R. (1932) “A technique for the measurement of attitudes”. Arch Psychol 1-55.

Minayo, M.C.S. (2007) "O desafio do conhecimento: Pesquisa qualitativa em saúde”. 10.ed. São Paulo.

Nielsen, Jakob. (2014) "Usability 101: Introduction to usability". Disponível em: $<$ http://www.useit.com/alertbox/20030825.html $>$.

Sancho-Asensio, A., Sole, X., Montero, J., Navarro, J., Canaleta, X., Vernet, D. (2014) "Support tool for the formation of working groups in collaborative learning environments", In: Information Systems And Technologies (Cisti), 2014 9th Iberian Conference On.

Satyanarayanan, M. (2001) "Pervasive Computing: vision and challenges", IEEE Personal Communications, [S.1.], v. 8, p. 10-17.

Vaughan-Nichols, S. (2009) "Will mobile computing's future be location, location, location?", Computer, 42(2):14 -17.

Wagner, A.; Barbosa, J.L.V.; Barbosa, D.N.F. (2014) "A Model for Profile Management applied to Ubiquitous Learning environments". Expert System with Application, v.41, n.4, p. 2023-2034

Weiser, M. (1991) "The computer for the 21st century", Scientific American, v. 265, n. 3, p. 94-104.

Yoon C, Kim S (2007) "Convenience and TAM in a ubiquitous computing environment: the case of wireless LAN". Electron Commer Res Appl 102-112. doi:10.1016/j.elerap.2006.06.009 